\title{
Da condição de cuidadores e do ato de cuidar de pessoas com deficiência intelectual
}

\section{The condition of caregivers and the act of caring for people with intellectual disabilities}

\author{
Rosane Seeger da Silva ${ }^{1}$, Fernanda dos Santos Pascotini ${ }^{2}$, Elenir Fedosse ${ }^{3}$ \\ http://dx.doi.org/10.11606/issn.2238-6149.v30i3p167-173
}

Silva RS, Pascotini FS, Fedosse E. Da condição de cuidadores e do ato de cuidar de pessoas com deficiência intelectual. Rev Ter Ocup Univ São Paulo. 2020 set.-dez.;30(3):167-73.

RESUMO: Introdução: A deficiência intelectual é uma interrupção no desenvolvimento ou uma formação incompleta da mente, causando, limitações no funcionamento, tanto intelectual e adaptativo da pessoa, originando-se antes dos 18 anos. Objetivo: Caracterizar cuidadores e seus atos de cuidar de pessoas com deficiência intelectual. Metodologia: Estudo censitário e descritivo, com abordagem quantitativa, realizado em um município de pequeno porte do interior gaúcho. Os dados foram coletados por meio de um instrumento, elaborado exclusivamente para os fins deste estudo, contendo questões fechadas referentes à caracterização dos cuidadores e das condições do ato de cuidar. Resultados: Foram encontrados 75 cuidadores de pessoas com deficiência intelectual, com uma média de idade de 51,56 anos, na maioria mulheres, mães e com baixa escolarização. Quanto aos principais cuidados prestados à pessoa com deficiência intelectual, destacam-se o auxílio com a administração de medicamentos, os cuidados com finanças e correspondências e na compra de alimentos e vestiários. Conclusão: As pessoas com deficiência intelectual estão sendo cuidadas, em sua maioria, por cuidadores informais, mulheres, com baixo nível de escolaridade, que se dedicam ao cuidado em tempo integral, não encontrando dificuldades, apesar de passarem por momentos de solidão e preocupações.

PALAVRAS-CHAVE: Cuidador; Deficiência intelectual; Família
Silva RS, Pascotini FS, Fedosse E. The condition of caregivers and the act of caring for people with intellectual disabilities. Rev Ter Ocup Univ São Paulo. Rev Ter Ocup Univ São Paulo. 2020 Sept.-Dec.;30(3):167-73.

ABSTRACT: Introduction: Intellectual disability is an interruption in development or an incomplete formation of the mind, causing limitations in functioning, both intellectual and adaptive of the person, originating before the age of 18. Objective: To characterize caregivers and their acts of caring for people with intellectual disabilities. Methodology: Census and descriptive study, with a quantitative approach, carried out in a small city in the interior of Rio Grande do Sul. Data were collected using an instrument, developed exclusively for the purposes of this study, containing closed questions regarding the characterization of caregivers and the conditions of the act of caring. Results: 75 caregivers of people with intellectual disabilities were found, with an average age of 51.56 years, mostly women, mothers and with low education. As for the main care provided to people with intellectual disabilities, the most important are assistance with medication administration, care with finance and correspondence and the purchase of food and changing rooms. Conclusion: Most people with intellectual disabilities are being cared for by informal caregivers, women, with a low level of education, who dedicate themselves to full-time care, not encountering difficulties, despite experiencing moments of loneliness and worries.

KEYWORDS: Caregiver; Intellectual deficit; Family

Este estudo resulta da dissertação de mestrado em Gerontologia intitulada "O processo de envelhecimento de pessoas com deficiência intelectual e sua família".

1. Universidade Federal de Santa Maria, Departamento de Fonoaudiologia, Programa de Pós-Graduação em Distúrbios da Comunicação Humana. Orcid: http://orcid.org/0000-0001-7980-4762. E-mail: rosane.seeger@hotmail.com.

2. Universidade Federal de Santa Maria, Departamento de Fonoaudiologia, Programa de Pós-Graduação em Distúrbios da Comunicação Humana. Orcid: http://orcid.org/0000-0001-6244-2795. E-mail: fepascotini@hotmail.com.

3. Universidade Federal de Santa Maria, Departamento de Fonoaudiologia, Programa de Pós-Graduação em Distúrbios da Comunicação Humana. Orcid: http://orcid.org/0000-0002-9691-7491. E-mail: efedosse@gmail.com.

Endereço para correspondência: Rosane Seeger da Silva. Rua Becker Pinto, 232 - Apto 402, Bairro Dores. Santa Maria, RS CEP: $97050-070$. 
Silva RS, et al. O ato de cuidar de pessoas com deficiência intelectual. Rev Ter Ocup Univ São Paulo. 2020 set./dez.;30(3):167-73.

\section{INTRODUÇÃO}

$\mathrm{A}$ deficiência intelectual (DI) é uma interrupção no desenvolvimento ou uma formação incompleta da mente, causando, limitações no funcionamento, tanto intelectual e adaptativo da pessoa, originando-se antes dos $18 \operatorname{anos}^{1}$. Pode ser causada por vários fatores, entre eles figuram os que atuam antes da concepção, os de ordem genética, ambientais, perinatais, pós-natais, nutricionais e, ainda, causas desconhecidas ${ }^{2}$.

Para que se possa caracterizar uma pessoa com DI, pelo menos, duas das áreas do comportamento adaptativo, que seguem, podem estar comprometidas: comunicação, autocuidado, vida no lar, interação social, saúde e segurança, uso de recursos da comunidade, autodeterminação, funções acadêmicas, lazer e trabalho ${ }^{1}$. A avaliação deve considerar fatores multidimensionais, tais como os ambientais e os pessoais em sua relação dinâmica, a fim de que se possa ofertar apoios específicos à aprendizagem e ao desenvolvimento da pessoa nas áreas que enfrentam dificuldades ${ }^{3}$.

Dados do Censo de 2010 indicam que 1,4\% da população brasileira apresenta DI. No Rio Grande do Sul, estado onde se localiza o município estudado, encontra-se a proporção de $0,7 \%$ de pessoas com $\mathrm{DI}^{4}$. Grande parte das pessoas com DI sofrem preconceitos e são consideradas como aquelas que necessitam de ajuda para existir; sendo representadas pelos seus familiares ou responsáveis legais, vivendo isoladas em suas casas ou em instituições especializadas, sem autonomia para suas necessidades ou interesses ${ }^{5}$. Neste contexto, assume fundamental importância o cuidador, aquele presente para auxiliar a pessoa com DI em todas as fases da vida.

De acordo com a Resolução da Diretoria Colegiada - RDC/ANVISA n ${ }^{\circ} 11$, de 26 de janeiro de 2006, o cuidador é "a pessoa com ou sem vínculo familiar capacitada para auxiliar o paciente em suas necessidades e atividades da vida cotidiana" (p. 2) ${ }^{6}$. Entende-se como cuidador formal a pessoa contratada financeiramente pelo paciente e/ou familiares para realizar o cuidado. Cuidadores informais são familiares, pessoas amigas, vizinhos ou voluntários que assumem o ato de cuidar sem preparo técnico e sem remuneração ${ }^{6}$.

No Brasil, são escassos os estudos sobre os cuidadores de pessoas com DI. Por isso, a necessidade de conhecer os cuidadores e o ato de cuidar de pessoas com DI, tendo em vista possíveis intervenções no campo da Saúde e da Educação para melhorar a qualidade de vida do cuidador e da pessoa com DI. Diante do exposto, suscitaram as seguintes indagações: quem são os cuidadores de pessoas com DI e como se dá o ato de cuidar destas pessoas? E, assim, este estudo teve como objetivo caracterizar o cuidador e o ato de cuidar da pessoa com DI em um município de pequeno porte do interior gaúcho.

\section{METODOLOGIA}

Este estudo resulta da dissertação de mestrado intitulada "O processo de envelhecimento de pessoas com deficiência intelectual e sua família". Trata-se de um estudo censitário, descritivo, com abordagem quantitativa, realizada em um município com aproximadamente 16 mil habitantes.

Ressalta-se que esta pesquisa foi realizada após ser aprovada pelo Comitê de Ética em Pesquisa da Universidade Federal de Santa Maria, parecer número 41459315.8.0000.5346, em 10 de março de 2015. Assim, foram cumpridas integralmente as disposições éticas estabelecidas pela resolução 466 de 12 de dezembro de 2012, do Conselho Nacional de Saúde 7 . Todos os participantes foram devidamente esclarecidos e orientados sobre a natureza, o objetivo, o caráter estritamente voluntário e não invasivo do estudo por ocasião do convite para participar, assinando o Termo de Consentimento Livre e Esclarecido (TCLE), em duas vias.

Foram encontrados 75 familiares/cuidadores, adultos, de ambos os sexos, que prestavam cuidados cotidianos a pessoas com DI. O contato com os sujeitos foi possibilitado através da busca ativa em instituições do município (Associação de Pais e Amigos de Excepcionais - APAE, Secretária Municipal de Educação, escolas municipais e estaduais, Centro de Atendimento Psicossocial - CAPS I e Instituição de Longa Permanência para Idosos - ILPI), locais estes que prestam algum tipo de atendimento a pessoas com DI no município investigado.

A coleta de dados foi feita por uma das autoras/ pesquisadora, nas residências dos sujeitos ou nas instituições, entre os meses de abril a setembro de 2015 , por meio da aplicação de um instrumento previamente elaborado para a coleta de dados. O instrumento continha perguntas fechadas, de fácil aplicação e análise. Ressalta-se que constituíram as variáveis do estudo as seguintes informações: características sociodemográficas (sexo, idade, estado civil, grau de parentesco, escolaridade e religião) e questões referentes a caracterização dos cuidados realizados pelos cuidadores, relações familiares, cuidados prestados, bem como, os sentimentos despertados em relação a responsabilidade no ato de cuidar de pessoas com DI. Foi elaborado diante de fundamentação na literatura, na experiência e no contato profissional das autoras com a população estudada. Para aplicação do instrumento, o tempo médio gasto foi de 50 minutos; não foi estabelecido limite de tempo pela autora/pesquisadora.

A organização do banco de dados foi realizada através do programa de computador Microsoft Excel, versão 2013 e 
a interpretação dos dados deu-se por da análise quantitativa e descritiva. Para melhor compreensão, os resultados são apresentados em tabelas demonstrados através de números absolutos (n) e em percentuais (\%).

\section{RESULTADOS}

Apresenta-se, na Tabela 1, as características dos 75 participantes da pesquisa:

Tabela 1 - Caracterização dos familiares/cuidadores de pessoas com DI quanto ao sexo, idade, estado civil, grau de parentesco, escolaridade e religião de um município do interior, $2015(\mathrm{n}=75)$

\begin{tabular}{|c|c|c|c|}
\hline Variáveis & Condição & $\mathbf{n}$ & $\%$ \\
\hline \multirow{2}{*}{ Sexo } & Masculino & 13 & 17,33 \\
\hline & Feminino & 62 & 82,66 \\
\hline \multirow{3}{*}{ Idade } & $20-40$ anos & 15 & 20 \\
\hline & 40-60anos & 43 & 57,33 \\
\hline & $>60$ anos & 17 & 22,67 \\
\hline \multirow{4}{*}{ Estado civil } & Solteiro & 16 & 21,33 \\
\hline & Casado & 35 & 46,66 \\
\hline & Separado & 08 & 10,67 \\
\hline & Viúvo & 16 & 21,33 \\
\hline \multirow{8}{*}{$\begin{array}{l}\text { Grau de } \\
\text { parentesco }\end{array}$} & Mãe & 46 & 61,33 \\
\hline & Irmãos & 09 & 12 \\
\hline & $\begin{array}{l}\text { Companheiro/ } \\
\text { esposo }\end{array}$ & 07 & 9,33 \\
\hline & $\begin{array}{l}\text { Nenhum } \\
\text { parentesco }\end{array}$ & 04 & 5,33 \\
\hline & Pai & 03 & 4 \\
\hline & Tios & 03 & 4 \\
\hline & Vó & 02 & 2,67 \\
\hline & Filho & 01 & 1,33 \\
\hline \multirow{6}{*}{ Escolaridade } & $\begin{array}{l}\text { Ensino } \\
\text { Fundamental } \\
\text { Incompleto }\end{array}$ & 47 & 62,66 \\
\hline & $\begin{array}{l}\text { Ensino } \\
\text { Fundamental } \\
\text { Completo }\end{array}$ & 10 & 13,33 \\
\hline & $\begin{array}{l}\text { Ensino Médio } \\
\text { Completo }\end{array}$ & 08 & 10,67 \\
\hline & $\begin{array}{l}\text { Ensino Médio } \\
\text { Incompleto }\end{array}$ & 01 & 1,33 \\
\hline & $\begin{array}{l}\text { Ensino } \\
\text { Superior }\end{array}$ & 08 & 10,67 \\
\hline & Analfabeto & 01 & 1,33 \\
\hline \multirow{3}{*}{ Religião } & Católica & 55 & 73,33 \\
\hline & Evangélica & 17 & 22,66 \\
\hline & Espírita & 03 & 4 \\
\hline
\end{tabular}

Fonte: Dados da pesquisa, 2015.
Quanto aos principais cuidados prestados à pessoa com DI pelos cuidadores, destacam-se o auxílio em atividades sociais e de cunho pessoal, conforme Tabela 2.

Tabela 2 - Caracterização dos cuidados realizados por familiares/ cuidadores à pessoa com DI, de um município do interior, 2015 $(\mathrm{n}=75)$

\begin{tabular}{lcc}
\hline Principais cuidados realizados & $\mathbf{N}^{\mathbf{0}}$ & $\mathbf{\%}$ \\
\hline $\begin{array}{l}\text { Cuidados com as finanças e } \\
\text { correspondências }\end{array}$ & 70 & 93,33 \\
Compras & 70 & 93,33 \\
Tomar remédios & 69 & 92 \\
Sair de casa & 49 & 65,33 \\
Realizar trabalhos domésticos & 35 & 46,66 \\
Alimentação & 29 & 38,66 \\
Nada & 01 & 1,33 \\
\hline
\end{tabular}

Fonte: Dados da pesquisa, 2015.

Em relação ao cotidiano de cuidados exercitados pelos cuidadores de pessoas com DI, foi possível constatar que a maioria não apresentou dificuldades, confira na Tabela 3 .

Tabela 3 - Desenvolvimento das atividades de cuidado pelos familiares/cuidadores de pessoas com DI de um município do interior, $2015(\mathrm{n}=75)$

\begin{tabular}{lll}
\hline $\begin{array}{l}\text { Tem dificuldade de lidar com a pessoa } \\
\text { com deficiência intelectual }\end{array}$ & $\mathbf{N}^{\mathbf{0}}$ & $\mathbf{\%}$ \\
\hline Não & 56 & 74,66 \\
Sim & 19 & 25,33 \\
$\begin{array}{l}\text { Tempo de cuidado a pessoa com } \\
\text { deficiência intelectual }\end{array}$ & \\
Integral & 67 & 89,33 \\
Parcial & 08 & 10,66 \\
Realizou algum curso para atuar & & \\
como cuidador & & \\
Sim (Cuidador Formal) & 02 & 2,66 \\
Não (Cuidador Informal) & 73 & 97,33 \\
\hline
\end{tabular}

Fonte: Dados da pesquisa, 2015.

A DI causa impacto na vida dos cuidadores, levando-os a passarem por diversas reações em relação à sua responsabilidade no cuidar de pessoas com DI. Questionados sobre este fato, destacaram os sentimentos descritos na Tabela 4. 
Silva RS, et al. O ato de cuidar de pessoas com deficiência intelectual. Rev Ter Ocup Univ São Paulo. 2020 set./dez.;30(3):167-73.

Tabela 4 - Sentimentos dos familiares/cuidadores de pessoas com DI em relação ao cuidado, município do interior, 2015 ( $\mathrm{n}=75)$

\begin{tabular}{lcc}
\hline $\begin{array}{l}\text { Sentimentos em relação à } \\
\text { responsabilidade no cuidar }\end{array}$ & $\mathbf{N}^{\mathbf{0}}$ & $\mathbf{\%}$ \\
\hline Prazer e Satisfação & 65 & 86,66 \\
Solidão & 06 & 8 \\
Preocupação & 01 & 1,33 \\
Outros & 03 & 4 \\
\hline
\end{tabular}

Fonte: Dados da pesquisa, 2015.

\section{DISCUSSÃO}

Os dados levantados neste estudo nos permitiram retratar a condição dos cuidadores e o cotidiano do cuidado de pessoas com DI, residentes em um município de pequeno porte do interior gaúcho. Apesar de este estudo ser local, pode-se dizer que existem realidades semelhantes nos municípios de pequeno porte; situação esta que revela as dificuldades de os gestores municipais cumprirem as diretrizes nacionais relativas à Saúde e Educação.

O reconhecimento desta realidade - cuidado à pessoa com DI prestado quase que exclusivamente pela família pode suscitar alternativas para beneficiar a implementação de ações de Saúde e de Educação que permitam atender as demandas de saúde, educacionais e sociais das pessoas com DI e de seus cuidadores, visando melhorar a qualidade de vida dessas populações. Está posta a necessidade de os profissionais da Saúde e da Educação bem formados competentes acerca dos diferentes mecanismos de inclusão social - assessorarem os gestores com conhecimentos atualizados sobre a atenção a pessoas com DI.

Destaca-se, neste estudo, a quantidade elevada de mulheres desempenhando essa função $(82,66 \%)$, dado também encontrado em outras investigações atuais ${ }^{8,9}$ relativas a cuidadores de pessoas com DI. O predomínio do gênero feminino encontrado é fato comum; estudos ${ }^{9}$ reforçam que quem realiza as tarefas de cuidado na família é a mulher que, geral e culturalmente, tem como atribuição a organização da rotina familiar, o cuidado dos filhos e também dos enfermos.

No que tange ao grau de parentesco, as mães eram a maioria das responsáveis pela assistência, corroborando com outros estudos recentes 8 . A mãe tem um papel fundamental na prestação de cuidados ao longo da vida da pessoa com $\mathrm{DI}^{10}$, o que implica redução de funções fora do lar (não ingresso ou desistência do emprego). Também, foi possível verificar, neste inquérito, que $89,3 \%$ dos cuidadores exercem a função em tempo integral, estando de acordo com resultado de outros estudos ${ }^{11}$ relacionados a cuidadores de pessoas com DI.
No que se refere à faixa etária, a média de idade dos cuidadores foi de 51,56 anos, havendo domínio daqueles que apresentaram entre 41 e 60 anos $(57,33 \%)$, ou seja, é expressiva a quantidades de familiares/cuidadores com idade superior a 40 anos. Este fato pode estar ligado às mudanças que vem ocorrendo nas famílias e na sociedade. Flores $^{12}$ afirma que, desde o ingresso da mulher no mercado de trabalho, o número de integrantes das famílias vem reduzindo. Por outro lado, a expectativa de vida das pessoas brasileiras é crescente. Como consequência, temse o aumento do convívio intergeracional. Desse modo, os familiares apresentam maior longevidade e maior contato com as outras gerações, o que pode favorecer na decisão de quem irá cuidar da pessoa com DI.

Quanto ao estado civil dos cuidadores, constatou-se que $46,66 \%$ eram casados, seguidos de viúvos $21,33 \%$. No geral, boa parte dos pais tem um relacionamento estável, (ambiente familiar sem violência e/ou necessidades extremas de sobrevivência, manutenção de renda por emprego ou por Benefício de Prestação Continuada - BPC), apresentando condições mínimas para oferta de um ambiente favorável para o crescimento e desenvolvimento de seu filho com $\mathrm{DI}^{13}$. A situação conjugal dos pais apresenta um papel fundamental para o desenvolvimento geral dos filhos e, conforme Silva e Dessen (p. 133) ${ }^{14}$, "a família constitui o primeiro universo de relações sociais da criança, podendo proporcionar-lhe um ambiente de crescimento e desenvolvimento", especialmente em se tratando de pessoas com DI, as quais necessitam de atenção e cuidados específicos para se desenvolverem.

Em relação à escolaridade, a maioria dos cuidadores $(62,66 \%)$ possui ensino fundamental incompleto. Tal dado vai ao encontro de outras pesquisas, que encontraram o predomínio de baixa escolaridade de cuidadores ${ }^{15}$. Segundo Fonseca e Pena ${ }^{16}$ a baixa escolaridade dos cuidadores pode influenciar na qualidade da compreensão das orientações sobre o cuidado aconselhadas pela equipe de saúde da família. Do nosso ponto de vista, certamente que o nível de escolaridade influencia no cuidado cotidiano da pessoa com DI; porém há que se destacar a conveniência de as equipes profissionais (da Saúde e da Educação, quando existentes) aproximarem-se das realidades de cada cuidador de modo a favorecer o entendimento das condições do sujeito cuidado, além de nivelar as orientações de acordo com as condições de apreensão de conteúdo do cuidador. Em outras palavras, há que se estabelecer real diálogo/entendimento entre o que os profissionais propõem como terapêutica e a execução cotidiana desta pelos cuidadores.

No que tange à religião, os dados encontrados nesta pesquisa revelaram que a maioria dos cuidadores $(73,33 \%)$ eram católicos - dados próximos ao encontrado no último 
Silva RS, et al. O ato de cuidar de pessoas com deficiência intelectual. Rev Ter Ocup Univ São Paulo. 2020 set./dez.;30(3):167-73.

Censo Demográfico ${ }^{4}$ que revelou $64,6 \%$ de brasileiros da religião católica. Tal dado também foi encontrado em pesquisa realizada com cinco famílias responsáveis pelo cuidado de pessoas com DI profunda ${ }^{17}$. Outro estudo revelou que a religiosidade (espiritualidade) se coloca como apoio das mães para seus questionamentos e receios; é na espiritualidade que elas buscam ajuda para a aceitação e o enfrentamento dos desafios do dia a dia ${ }^{10}$. Conforme Santos ${ }^{18}$, as estratégias de enfrentamento pela religiosidade podem ser entendidas como desvio das dificuldades encontradas no cotidiano ou negação frente à realidade do problema. A busca de explicações advindas da religiosidade/espiritualidade podem ser as únicas formas de apoio que as mães encontram (no caso do referido autor).

No caso deste estudo, pode-se dizer que o apoio na religiosidade é um dos poucos recursos de que cuidadores dispõem no município, já que o acompanhamento profissional especializado é escasso (os recursos referentes a terapeutas como educadores especiais, fisioterapeutas, fonoaudiólogos, psicólogos e terapeutas ocupacionais, por exemplo, restringem-se a serviços filantrópicos que não conseguem absorver toda a demanda do município); os cuidadores quando acessam tais serviços é porque têm condições financeiras de buscá-los, no setor privado, em municípios da região.

Constatou-se, neste estudo, mais um fato discutido na literatura e bem característico da realidade de pessoas com DI: a dependência de familiares/cuidadores para realizar suas atividades cotidianas (Tabela 2). No contexto da pessoa DI e reconhecidamente pelos profissionais desta área, o cuidador tem papel imprescindível e decisivo ao longo da vida da pessoa; ele desempenha a função de auxiliá-la a fazer sua higiene, a tomar remédios, a realizar os tratamentos necessários, visando suas atividades de participação social e, por isso, facilitando sua independência nas atividades da vida diária (AVD), sua autonomia e privacidade. $\mathrm{Na}$ base do descrito, o cuidador dispensa afeto, carinho, escuta, compreensão e outras formas de apoio emocional à pessoa com DI ${ }^{19,20}$. Neste sentido, considera-se que o vínculo entre esses sujeitos - profissionais, familiares/cuidadores e pessoas com DI - é indispensável para que se alcance qualidade de vida dos envolvidos.

Pelos fatos acima, e também pela experiência das autoras deste estudo, perguntou-se aos cuidadores se tinham dificuldades na lida cotidiana das pessoas com DI. A maioria dos pesquisados relatou não apresentarem dificuldades, conforme demonstrado na Tabela 3. Tal fato pode ser interpretado em função de a maioria dos cuidadores serem mães; assim o cuidado é facilitado, já teoricamente, espera- se que as mães reconheçam, entendam e organizem a vida dos filhos, independentemente, de apresentarem ou não DI.

Além das mães, outros familiares prestavam cuidados à pessoa com DI alcançando-se o percentual de $97,3 \%$. Um fato relevante de ser discutido, pois estes cuidadores não realizaram formação técnica/curso para tal tarefa. Assim, esses cuidadores são informais, pois não possuem a formação específica e tampouco remuneração. Este dado está em acordo com outro estudo cuja consideração é a de que, normalmente, o cuidador informal é um membro da família ou da comunidade e que, infelizmente, a grande maioria dos cuidadores informais encontra-se sem informações e suporte adequado para assistir a pessoa sob seus cuidados ${ }^{21}$.

Do nosso ponto de vista, essa condição reitera a conveniência de vinculação efetiva entre os profissionais (especialmente os terapeutas, especialista da Saúde em desenvolvimento humano) e os familiares das pessoas com DI (cuidadores cotidianos). Tal implicação/vinculação efetiva pode resultar na real promoção da saúde, entendida como a condição de as pessoas conseguirem identificar os determinantes do processo saúde-doença e participarem de maneira transformadora em suas realidades ${ }^{22}$. Neste sentido, os cuidadores, devidamente apoiados pelos profissionais, poderiam reconhecer seus limites, suas necessidades, seus desafios e, assim, organizarem-se para exigir os serviços prioritários para garantia do direito humano à vida com qualidade.

Os sentimentos positivos foram os mais presentes nos cuidadores - o prazer e a satisfação $(86,66 \%)$, conforme mostra a Tabela 4. Este fato pode significar que o mais relevante é cuidar da pessoa querida, independentemente dos momentos difíceis e/ou estressantes. Gonçalves et al. ${ }^{23}$ afirmam que dentre os principais motivos que levam uma pessoa a cuidar estão os sentimentos de obrigação e gratidão, amparados por aspectos culturais e religiosos e/ou pela ausência de outras pessoas para a tarefa. Nessa perspectiva, a carga emocional envolvida na relação familiar, influencia diretamente na função de cuidar.

Cuidar não é fácil, pois exige muitas mudanças na vida de quem cuida e demanda a realização de atividades intensas, delicadas e, por vezes, sofridas. As atividades que são atribuídas ao cuidador, associadas à alteração na rotina $\mathrm{e}$ o tempo despendido ao cuidado, pode ter impacto negativo na qualidade de vida deste ${ }^{24}$. Portanto, associado ao fato de que o cuidador é, geralmente, uma pessoa que se encontra em processo de envelhecimento, pode-se inferir que ele tem um potencial para adoecimento e que sua capacidade funcional está constantemente em risco ${ }^{25}$.

Pelo aqui discutido, pode-se afirmar que é de suma importância que exista o acolhimento do cuidador; que os serviços possam ofertar conhecimentos que facilitem suas 
Silva RS, et al. O ato de cuidar de pessoas com deficiência intelectual. Rev Ter Ocup Univ São Paulo. 2020 set./dez.;30(3):167-73.

atividades, promovam a saúde e reduzam as limitações do ato de cuidar. Então, o cuidador descobrirá formas distintas para prestar cuidado, adaptando-se às demandas, sem anular suas próprias necessidades.

\section{CONCLUSÃO}

Os dados apresentados neste estudo permitiram conhecer os cuidadores e o ato de cuidar da pessoa com DI de um município de pequeno porte do interior gaúcho com restrita rede de atenção à saúde. De modo geral, constatouse que as pessoas com DI estão sendo cuidadas, em sua maioria, por cuidadores informais, mães, com baixo nível de escolaridade, que se dedicam ao cuidado em tempo integral. Além disso, evidenciou-se que os cuidadores não apresentam dificuldades importantes no cotidiano e que cuidam, principalmente, por prazer e satisfação.
No contexto da DI, convém que os cuidadores recebam apoio de profissionais qualificados da área da Saúde e da Educação para que possam aprimorar os cuidados, ampliar as perspectivas de independência e autonomia das pessoas com DI e, ainda, estabelecer estratégias para preservar a saúde física e emocional.

Como limitações do estudo pode-se apontar o fato de ter sido realizado em um pequeno município, ou seja, de refletir a situação de um local com pouca densidade populacional. No entanto, a realidade retratada pode estar refletindo características específicas dessa população em municípios de médio e grande porte. Neste sentido, seria interessante realizá-la em outros municípios e regiões, aumentando-se o número de cuidadores e, ainda, acrescentar uma entrevista para que se possa aprofundar as questões emocionais e vivenciais dos cuidadores de pessoas com DI.

Participação dos autores: Rosane Seeger da Silva: responsável pela pesquisa, concepção do manuscrito, redação e revisão do texto. Fernanda dos Santos Pascotini: responsável pela revisão do texto. Elenir Fedosse: orientadora da pesquisa, apoiou a elaboração e revisão do manuscrito. Todos os autores participaram da aprovação da versão final do artigo para publicação.

\section{REFERÊNCIAS}

1. Fierro A. Os alunos com deficiência mental. In: Coll C, Marchesi A, Palacios J. Desenvolvimento psicológico e educação: transtornos de desenvolvimento e necessidades educativas especiais. 2a ed. Porto Alegre: Artmed; 2004. p.193-214.

2. Assumpção Junior FB. Deficiência mental. In: Assumpção Junior FB; Tardivo, LSLPC. Fundamentos de psicologia: psicologia do excepcional: deficiência física, mental e sensorial. Rio de Janeiro: Guanabara Koogan; 2008. p.5-13.

3. Carvalho ENS, Carvalho RE, Costa SM. Público alvo. In: Carvalho ENS, Carvalho RE, Costa SM. Política de atenção integral e integrada para as pessoas com deficiência intelectual e múltipla. Brasília: Federação Nacional das APAEs; 2011.

4. Brasil. Instituto Brasileiro de Geografia e Estatística (IBGE). Censo demográfico 2010. Disponível em: http://www.ibge. gov.br/home/estatistica/populacao/censo2010/default.shtm.

5. Moraes MRQ, Souza DP. O idoso e a deficiência: uma análise da situação sócio afetiva dos alunos com deficiência intelectual em fase de envelhecimento. Rev Igapó Amazonas. 2009;1(1):18-27. Disponível em: http://www.ifam.edu.br/cms/ images/stories/arquivos/revista_igapo/edicao_03/artigo02.pdf.

6. Brasil. Ministério da Saúde. Agência Nacional de Vigilância Sanitária (ANVISA). RDC/ANVISA nº 11 , de 26 de janeiro de 2006. Dispõe sobre o regulamento técnico de funcionamento de serviços que prestam a atenção domiciliar. Disponível em: http://e-legis.anvisa. gov.br/leisref/public/showAct. php?id=20642\&word.

7. Brasil. Conselho Nacional de Saúde. Resolução n 466 , 2012. Diretrizes e normas regulamentadoras de pesquisa envolvendo seres humanos. Brasília, 13 jun. 2013. Seção 1 p. 59. Disponível em: http://www.conselho.saude.gov.br/ resolucoes/2012/Reso466.pdf

8. Demarco DA, Jardim VMR, Kantorski LP. Perfil dos familiares de usuários de Centros de Atenção Psicossocial: distribuição por tipo de serviço. Rev Fund Care. 2017;9(3):732-7. doi: http://dx.doi. org/10.9789/2175-5361.2017.v9i3.732-737.

9. Tomaz RVV, Santos VA, Avó LRS, Germano CMR, Melo DG. Impacto da deficiência intelectual moderada na dinâmica e na qualidade de vida familiar: um estudo clínicoqualitativo. Cad Saúde Pública. 2017;33(11):e00096016. https://doi.org/10.1590/0102-311x00096016.

10. Barbosa MAM, Chaud MN, Gomes MMF. Vivências de mães com um filho deficiente: um estudo fenomenológico. Acta Paul Enferm. 2008;21(1):46-52. https://doi.org/10.1590/ S0103-21002008000100007.

11. Ballarin MLGS, Benedito AC, Kron CA, Christovam D. Perfil sociodemográfico e sobrecarga de cuidadores 
informais de pacientes assistidos em ambulatório de terapia ocupacional. Cad Ter Ocup UFSCar. 2016;24(2):315-21. https://doi.org/10.4322/0104-4931.ctoAO0607.

12. Flores GC. "Eu cuido dela e ela me cuida": um estudo qualitativo sobre o cuidado intergeracional com o idoso [dissertação]. Santa Maria, RS: Centro de Ciências da Saúde, Universidade Federal de Santa Maria; 2008. Disponível em: http://repositorio.ufsm.br/handle/1/7307.

13. Silva RS, Fedosse E. Perfil sociodemográfico e qualidade de vida de cuidadores de pessoas com deficiência intelectual. Cad Bras Ter Ocup. UFSCar. 2018;26(2):357-66. https://doi. org/10.4322/2526-8910.ctoAO1220.

14. Silva NL, Dessen MA. Deficiência mental e família: implicações para o desenvolvimento da criança. Psicol Teor Pesq. 2001;17(2):133-41. https://doi.org/10.4322/ 2526-8910.ctoao 1220 .

15. Camara FSS, Martins WLL, Moura MLN, Melo CS, Medeiros NSR, Gadelha ECM, et al. Perfil do cuidador de pessoas com deficiência. Rev Bras Cienc Saúde. 2016;20(4):269-76. doi: 10.4034/RBCS.2016.20.04.02.

16. Fonseca NR, Penna AFG. Perfil do cuidador familiar do paciente com sequela de acidente vascular encefálico. Ciênc Saúde Coletiva. 2008;13(4):1175-80. https://dx.doi.org/10.1590/ S1413-81232008000400013.

17. Ferreira PR, Fiamenghi Jr GA. Relações familiares de cuidadores de pessoas com deficiência intelectual profunda. Pensando Fam. 2015;19(1):130-41. Disponível em http://pepsic.bvsalud.org/ scielo.php?script=sci_arttext\&pid=S1679-494X2015000100011.

18. Santos WJ. A religiosidade como estratégia de enfrentamento do processo de incapacidade funcional dos idosos da cidade de Bambuí, Minas Gerais [dissertação]. Belo Horizonte: Fundação Oswaldo Cruz. Centro de Pesquisas René Rachou, Laboratório de Educação em Saúde e Ambiente; 2012. Disponível em: https://www.arca.fiocruz.br/handle/icict/5497

19. Mazza MMPR, Lefèvre F. A instituição asilar segundo o cuidador familiar do idoso. Saúde Soc. 2004;13(3):68-77. https://dx.doi.org/10.1590/S0104-12902004000300008.

20. Ceschini M. Por que assistência domiciliar. In: Dias ELF, organizador. Orientações para cuidadores informais na assistência domiciliar. Campinas: UNICAMP; 2005.

21. Garrido R, Menezes PR. Impacto em cuidadores de idosos com demência atendidos em um serviço psicogeriátrico. Rev Saúde Pública. 2004;38(6):835-1. Hjttps://dx.doi.org/10.1590/ S0034-89102004000600012.

22. Brasil. Ministério da Saúde, Secretaria de Políticas de Saúde. Projeto promoção da saúde. As cartas de promoção da saúde. Brasília (DF): Ministério da Saúde; 2002.

23. Gonçalves LHT, Alvarez AM, Sena ELS, Santana LWS, Vicente FR. Perfil da família cuidadora de idoso doente/fragilizado do contexto sociocultural de Florianópolis, SC. Texto Contexto Enferm. 2006;15(4):570-77. https://doi.org/10.1590/ S0104-07072006000400004.

24. Amendola F, Oliveira MAC, Alvarenga MRM. Qualidade de vida dos cuidadores de pacientes dependentes no programa de saúde da família. Texto Contexto Enferm. 2008;17(2):266-72. doi: 10.1590/S0104-07072008000200007.

25. Karsch UM. Idosos dependentes: famílias e cuidadores. Cad Saúde Pública. 2003;19(3) 861-66. https://doi.org/10.1590/ S0102-311X2003000300019. 